\title{
Nivel de apropiación del Internet y nuevas tecnologías en las Pymes colombianas exportadoras o potencialmente exportadoras ${ }^{1}$
}

Fecha de recepción: 24 de marzo de 2011 Fecha de aprobación: 23 de mayo de 2011

\author{
Sandra Patricia Rojas Berrío \\ srojasbe@poligran.edu.co \\ Politécnico Grancolombiano
}

Administradora de empresas y magíster en Administración. Directora del Departamento de Investigación, Desarrollo e Innovación de la Fundación Politécnico Grancolombiano.

\section{Ricardo Arturo Vega Rodríguez \\ rvegarod@poli.edu.co \\ Politécnico Grancolombiano}

Químico farmacéutico y magíster en Administración. Profesor e investigador del área de Mercadeo de la Fundación Politécnico Grancolombiano.

\section{Resumen}

Colombia, según el Índice Global de Competitividad, se ubica en el puesto 68 (DNP, 2010) y se encuentra en la posición 51 de desarrollo tecnológico del mundo. Sin embargo, paradójicamente creció de 2009 a 2010 tres lugares en preparación tecnológica y decreció uno en sofisticación de los negocios.

Dado lo anterior, el propósito del trabajo que se presenta fue el de identificar las formas en las que las Pymes exportadoras o potencialmente exportadoras usaban tecnologías de información y comuni-

\section{Abstract}

Colombia ranks 68th in the Global Index of Competitiveness (DNP, 2010) and 51st within the technological development of the world. However, paradoxically, it moved upward three places from 2009 to 2010 in technological training and went down one place in business sophistication.

Because of this, the purpose of this article is to identify the way exporting or potentially exporting SMEs used information and communication technologies related to the Internet. This is to check the level of technology adoption and its in-

1 Los autores agradecen la colaboración en este trabajo a Eric Julián Rodríguez Martínez estudiante de Ingeniería Industrial de la Fundación Politécnico Grancolombiano. 
caciones asociadas con el Internet. El fin de lo anterior, es revisar el nivel de adopción de la tecnología y la incidencia de ello en su potencial de exportación con miras a revisar su adaptación al cambio.

\section{Palabras clave}

Internet y nuevas tecnologías, Pymes exportadoras o potencialmente exportadoras, nivel de apropiación. cidence in the exporting potential, as well as its change adaption.

\section{Keywords}

Internet and New Technologies, Exporting or Potentially Exporting SMEs, Appropriation Level 


\section{Introducción}

La internacionalización es una de las acciones organizacionales más importantes y generalizadas que ha cambiado el entorno competitivo de los negocios, pues ha permitido abrir los mercados nacionales a nuevos competidores, a la vez que ha creado nuevas oportunidades de negocio para empresas pequeñas y grandes (Grant, 2004). Ha traído consigo además, un mayor conocimiento de mercadeo y también una disminución de la incertidumbre (Moen \& Servais, 2002), a través de la participación gradual de las empresas a nivel internacional. También es uno de los factores que hacen reconocidos los productos y servicios de un país en otras latitudes y es por ello que se escogen para este estudio las empresas exportadoras o potencialmente exportadoras.

Adicionalmente, una de las maneras por medio de la cual es posible medir la competitividad de un país o una región, es la capacidad que tienen sus organizaciones de adaptarse a los cambios del mercado en la cual se desenvuelven (Armario, Horrillo, \& Robles, 2009). Uno de los factores que han cambiado la manera en la cual las organizaciones en todo el mundo ha sido el Internet, pues gracias a él se encuentran formas de divulgar el portafolio de productos y servicios, realizar transacciones tanto con los proveedores como con los clientes e interactuar y ser capaz de responder a quejas, peticiones, reclamos y trámites.

Colombia según el Índice Global de Competitividad se ubica en el puesto 68 (DNP, 2010) y se encuentra en la posición 51 de desarrollo tecnológico del mundo. Sin embargo, paradójicamente creció de 2009 a 2010 tres lugares en preparación tecnológica y decreció uno en sofisticación de los negocios.

Dado lo anterior, el propósito del trabajo que se presenta fue el de identificar las formas en las que las Pymes exportadoras o potencialmente exportadoras usaban tecnologías de información y comunicaciones asociadas con el Internet. El fin de lo anterior, es revisar el nivel de adopción de la tecnología y la incidencia de ello en su potencial de exportación, con miras a revisar su adaptación al cambio.

La metodología que se utilizó fue una investigación no experimental de tipo descriptivo. La herramienta de captura utilizada fueron encuestas vía Internet por medio del aplicativo de encuestafacil. Adicionalmente, se hizo un refuerzo vía entrevista personal con apoyo de estudiantes de la institución donde trabajan los autores y provenientes de las diferentes regiones del país, que fueron parte de la muestra.

Los capítulos de la encuesta contemplaron las temáticas: caracterización de la empresa, evaluación de Internet y evaluación de nuevas tecnologías (hardware y software). El primer capítulo tuvo una pretensión demográfica. En contraste, el segundo revisó aspectos como los relativos a servicio utilizado y consumo de Internet, comportamiento del sitio web, percepción y opinión sobre Internet y uso del mismo como herramienta de comunicación, de gestión y de marketing. Finalmente, el apartado evaluación de nuevas tecnologías (hardware y software) indagó acerca de la situación actual de adquisición de dichas herramientas y su potencial.

La muestra fue compuesta por 1000 Pymes con calidad exportadora o potencialmente exportadora y fue obtenida a 
partir de un muestreo estratificado proporcional con respecto al tamaño de la población de las principales ciudades de Colombia distribuida así: Bogotá 49\%, Cali y Medellín 16\%, Bucaramanga, Pereira, Neiva, Ibagué, Santa Marta, Barranquilla y Cartagena (35\%).

Como principales hallazgos se encontró que el $60 \%$ de las empresas encuestadas utiliza el Internet para publicar sus promociones, descuentos e incentivos, mientras que el $40 \%$ no opta por esta opción. De los que utilizan este medio, el $80 \%$ tiene conocimiento del posicionamiento de marketing de su empresa en Internet, mientras que el $20 \%$ no realiza un seguimiento de éste. Una gran mayoría de encuestadas se interesa en la cantidad de visitantes que recibe a diario para saber cuáles pueden ser posibles clientes, cómo retener a los actuales y recuperar los perdidos. El $64 \%$ de las empresas no realiza mercadeo electrónico, mientras que un $36 \%$ sí lo hace.

Un hallazgo contradictorio es que tan sólo el $61 \%$ de las empresas posee página web y, entendiendo que la muestra estaba compuesta por empresas exportadoras o potencialmente exportadoras, es un tema preocupante dado que una de las herramientas de visibilidad en el mundo es la página web.

En el país se están impulsando regionalmente unos comités denominados Universidad-Empresa-Estado; entre sus misiones está generar spin-off que apunten al desarrollo del país e impulsar el desarrollo tecnológico. Una de las recomendaciones del presente trabajo para dichas organizaciones, es revisar más de cerca el nivel de apropiación de las herramientas tecnológicas en las Pymes con el fin de que mejoren su productividad y la comunicación de sus productos.
Algunas de las páginas de las organizaciones indagadas sólo manifiestan la misión y la visión de las empresas y no reportan información precisa al potencial consumidor con respecto a portafolio de productos y servicios. Luego, una de las recomendaciones para las organizaciones es explotar el potencial transaccional de las herramientas tecnológicas para contribuir a la gestión de relaciones con los clientes.

De igual modo, es recomendable que la actividad de actualización tecnológica sea permanente y se convierta en un tema estratégico que permita no sólo impulsar la productividad sino que se torne en una ventaja competitiva. Para ello, es importante trabajar internamente en la organización en temáticas como adaptación al cambio y gestión del conocimiento.

En conclusión, los anteriores resultados posibilitan un mejor entendimiento de la dinámica de las tecnologías de información y comunicaciones en las Pymes en Colombia, que denotan un significativo atraso en la apropiación y utilización de herramientas de gestión que podrían contribuir al alcance de las metas de las compañías.

\section{Marco teórico}

Las tecnologías de información y comunicaciones (TIC) han tenido un nivel de avance, desarrollo y apropiación vertiginoso a nivel de hogares y empresas. En estas últimas, la necesidad de mejorar sus procesos de gestión y comunicación hace necesaria la apropiación de dichas herramientas. Lo anterior, en razón de las crecientes presiones en términos competitivos que hacen de Internet una 
herramienta estratégica (Porter, 2001; Ching \& Ellis, 2004; Camisón \& López, 2010) que impacta procesos de comunicación, control y colaboración.

El hecho de no usar adecuadamente las TIC o tener temor de hacerlo, no es tan grave para una empresa grande o incluso multinacional puesto que las pérdidas que pueda generar ello pueden ser compensadas por sus subsidiarias o filiales. Adicionalmente, aquellas de vocación local pueden tener capitales para soportar dichas carencias. En contraste, el no estar "al día" en el caso de las Pymes, podría significar incluso el cierre del negocio (Buckley, 1989 y Welsh \& White, 1981).

En la literatura revisada, se encontraron estudios acerca del uso de software libre en MiPymes (Martinez, 2009) en el contexto colombiano y en el uso de las TIC para el mejoramiento de la gestión de empresas del mismo tamaño en sectores productivos chilenos (Velásquez, Fuentes \& Jiménez, 2007).

Por un lado, en el primer artículo, el mencionado autor reflexiona acerca de las posibilidades que deberían tener las $\mathrm{Mi}$ Pymes para acceder a la información sobre estas herramientas, para así experimentar con ellas y explorar las posibilidades de hacer su gestión más eficiente.

Por otro, el estudio chileno evalúa la situación de las MiPymes con respecto al uso de las TIC y revisa la factibilidad de usar soluciones como el software libre para la operación diaria en los sectores acuícola, forestal y frutícola.

Otro estudio hallado y relativo al tema, se enfoca en conceptualizar las percepciones del riesgo y sus implicaciones para las MiPymes activas en internacionalización online (Pezderka \& Sinkovics, 2010). En él, los autores se concentran en el rol que tienen las TIC en la internacionalización para las MiPymes y en desarrollar una propuesta que parte del marco de Dunning (2001) que enfocan a la percepción de riesgo.

Adicionalmente, ningún estudio en el campo colombiano ha revisado a profundidad el nivel apropiación del Internet y nuevas tecnologías en las Pymes colombianas exportadoras o potencialmente exportadoras.

\section{Metodología}

La pregunta de investigación que motivó esta investigación fue: ¿cuál es nivel de apropiación del Internet y las nuevas tecnologías en las Pymes colombianas exportadoras o potencialmente exportadoras? La gráfica a continuación muestra el proceso de investigación llevado a cabo.

Gráfica 1. Proceso de investigación nivel de apropiación del Internet y las nuevas tecnologías en las

Pymes colombianas exportadoras o potencialmente exportadoras.

\begin{tabular}{|l|}
\hline Diseño de instrumentos de captura de información \\
\hline - 190 variables \\
\hline Recolección de información \\
\hline $\begin{array}{l}\text { - Cuestionario web (refuerzo telefónico } \\
\text { y personal) }\end{array}$ \\
\hline Depuración de la información \\
\hline - 1220 encuestas (740 válidas) \\
\hline Análisis de información \\
\hline - Análsis de conglomerados \\
- Análsis descriptivo \\
\hline
\end{tabular}




\section{Diseño de instrumento de captura de información}

Durante el diseño se contó con la participación de dos académicos y dos empresarios quienes ayudaron a construir y validar el instrumento. Se formularon preguntas según tres áreas de interés para el desarrollo de la investigación: caracterización de la empresa, evaluación de Internet y evaluación de nuevas tecnologías (hardware y software).

El capítulo de caracterización de la empresa fue diseñado para recolectar datos demográficos y de verificación de cumplimiento de las necesidades muestrales. En dicho capítulo el encuestado fue indagado acerca de aspectos como: razón social, dirección de la compañía, teléfono, fax, correo electrónico, ciudad del domicilio principal de la empresa, número de sucursales, número de empleados, ingresos anuales, intención o acción de exportación en el último año, porcentaje de ingresos que representa el anterior concepto en la organización, zonas geográficas a las que ha exportado o planea hacerlo, actividad económica y tiempo en la misma.

En el capítulo de evaluación de Internet, se evaluaron las siguientes variables: aspectos relativos al consumo y servicio, sitio web (apariencia, existencia y usos), percepción y opinión sobre Internet y uso como herramienta de gestión comercial (Marketing, ventas, promoción y publicidad). Finalmente, en el capítulo de evaluación de nuevas tecnologías (hardware y software), se indagó sobre la actualización en términos de hardware y software.

\section{Recolección y depuración de la información}

Se diseñó el formulario previamente validado por la empresa EncuestaFácil, expertos en el aplicativo web, y se hicieron tres olas de envíos durante dos meses a una base de datos de Pymes en Colombia con cerca de diez mil registros. Durante los envíos se hizo un muestreo estratificado con afijación proporcional según las ciudades (estratos) que más empresas de dicho tamaño tenían en el marco muestral; con esa selección se procedió a hacer refuerzo telefónico durante el primer mes y personal durante el segundo.

El anterior proceso llevó a obtener una tasa de respuesta bruta de 12,2\%. Sin embargo, al depurar la información, se encontró que sólo 740 registros cumplían con la característica de ser una pequeña o mediana empresa, exportadora o potencialmente exportadora, obteniendo así una tasa de respuesta neta de 7,4\%. La información que se usa para el análisis en esta investigación se distribuye por ciudad principal de la siguiente manera:

Tabla 1. Distribución de la muestra por ciudad.

\begin{tabular}{|l|r|r|r|r|}
\hline \multicolumn{5}{|c|}{ EN QUÉ CIUDAD SE ENCUENTRA EL DOMICILIO PRINCIPAL DE SU EMPRESA } \\
\hline \multicolumn{1}{|c|}{ Ciudad } & Frecuencia & Porcentaje & Porcentaje válido & Porcentaje acumulado \\
\hline - Bogotá & 275 & 37,2 & 37,2 & 37,2 \\
\hline - Cali & 79 & 10,7 & 10,7 & 47,8 \\
\hline - Medellín & 137 & 18,5 & 18,5 & 66,4 \\
\hline - Pereira & 51 & 6,9 & 6,9 & 73,2 \\
\hline - Neiva & 41 & 5,5 & 5,5 & 78,8 \\
\hline
\end{tabular}




\begin{tabular}{|l|r|r|r|r|}
\hline \multicolumn{5}{|c|}{ EN QUÉ CIUDAD SE ENCUENTRA EL DOMICILIO PRINCIPAL DE SU EMPRESA } \\
\hline - Ibagué & 42 & 5,7 & 5,7 & 84,5 \\
\hline - Bucaramanga & 24 & 3,2 & 3,2 & 87,7 \\
\hline - Santa Marta & 9 & 1,2 & 1,2 & 88,9 \\
\hline - Barranquilla & 51 & 6,9 & 6,9 & 95,8 \\
\hline - Cartagena & 27 & 3,6 & 3,6 & 99,5 \\
\hline - Pasto & 4 &, 5 &, 5 & 100,0 \\
\hline \multicolumn{1}{|r|}{ Total } & 740 & 100,0 & 100,0 & \\
\hline
\end{tabular}

\section{Análisis de información}

El paquete estadístico utilizado para analizar la información fue PAWS Statistics, anteriormente denominado SPSS, en donde, posterior al proceso de depuración de la información, se procedió a realizar un análisis descriptivo y luego de conglomerados bietápicos con el fin de hallar diferenciales entre la muestra de empresas.

\section{Hallazgos}

En la primera parte de esta sección se revisarán los resultados de la investigación de una forma descriptiva. Posteriormente, se mostrarán hallazgos de carácter interpretativo dado que el procesamiento estadístico llevado a cabo permitió hallar dos conglomerados cuyas diferencias y similitudes fueron analizadas por medio de las pruebas estadísticas que ofrece SPSS para medias de dos muestras independientes.

\subsection{Análisis descriptivo}

Al revisar aspectos como el tipo de conexión a Internet que es la más utilizada por las Pymes en Colombia, se encuentra que el ADSL tiene un 29,1\%, seguido del Cable Modem con un 20,9\% y la Fibra Óptica con un 18,5\% de utilización. Este resultado se puede contrastar con los hallazgos de una de las preguntas que revisó el uso de sistemas de videoconferencia para comunicarse con clientes y proveedores en el exterior, dado que nos muestra que un $81,1 \%$ de las empresas no las realizan y tan sólo un 18,9\% de las empresas sí lo hacen.

Por otro lado, indagando el aspecto de si la empresa posee una página web, se encuentra que tan sólo el 53\% de los encuestados poseen esta alternativa de comunicación. Adicionalmente, la información recolectada evidencia que el contenido de los sitios de la gran mayoría de las organizaciones encuestadas tienen un enfoque informacional $(68,9 \%)$ más que transaccional. En contraste, las que la poseen reconocen las ventajas de tenerla y la utilizan de distintas maneras con el fin de darse a conocer más y aumentar las ventas. Lamentablemente, un bajo porcentaje de las empresas utilizan dos idiomas entre los cuales uno ellos es el español y el otro es el inglés seguido del portugués. La siguiente tabla muestra los resultados.

Tabla 2. Número de idiomas disponibles en su Página Web.

\begin{tabular}{|l|c|c|}
\hline \multicolumn{2}{|c|}{$\begin{array}{c}\text { NÚMERO DE IDIOMAS } \\
\text { EN LA PÁGINA WEB }\end{array}$} & PORCENTAJE \\
\hline Válidos & Uno & 35,3 \\
\cline { 2 - 3 } & Dos & 20,3 \\
\cline { 2 - 3 } & Mas & 1,6 \\
\cline { 2 - 3 } & Total & 57,2 \\
\hline Total & 100,0 \\
\hline
\end{tabular}


$\mathrm{Al}$ respecto de los usos de Internet, se encontró que dicha herramienta es dedicada para actividades como comunicación con proveedores e intercambio de archivos principalmente. En contraste, al indagar sobre el uso de Internet como herramienta de gestión de mercadeo se encuentra que un $45,4 \%$ de las empresas le utiliza para hacer publicidad, mientras que un $32,8 \%$ no. Adicionalmente, los encuestados manifestaron que un $42 \%$ le usa para publicar las promociones, incentivos y descuentos de sus productos. Por otro lado, un $58,7 \%$ de empresas que sí le usan para realizar investigación de mercados y un $41,3 \%$ de empresas que nunca han utilizado Internet para hacerlo. Además, un $81 \%$ de empresas nunca ha utilizado Internet para realizar ventas, argumentando razones como falta de conocimiento y falta de herramientas tecnológicas. La tabla a continuación evidencia un tema relacionado con los anteriores y que podría resumir el nivel de apropiación de las nuevas tecnologías y el Internet como herramienta de gestión de mercadeo:

Tabla 3. ¿Dispone su organización de un sistema informático en línea para CRM (gestión de relaciones con los clientes)?

\begin{tabular}{|l|l|c|}
\hline \multicolumn{2}{|c|}{} & PORCENTAJE \\
\hline \multirow{3}{*}{ Válidos } & Si & 8,8 \\
\cline { 2 - 3 } & No & 64,6 \\
\cline { 2 - 3 } & Total & 73,4 \\
\hline Perdidos & Sistema & 26,6 \\
\hline Total & 100,0 \\
\hline
\end{tabular}

En los temas de actividades relacionadas con exportación, más del 30\% de las empresas le utiliza menos del $20 \%$ de Internet para dichas actividades, seguido de un $21,2 \%$ de empresas que utiliza del
$75 \%$ al $100 \%$ y un $12 \%$ de empresas que no utiliza para nada Internet en sus actividades de exportación. Adicionalmente, las que sí la utilizan lo hacen para actividades como pagos, giros y facturación y comunicación y soporte a clientes.

Tabla 4. ¿Qué porcentaje de uso tiene Internet para sus actividades de exportación?

\begin{tabular}{|l|l|c|}
\hline \multicolumn{2}{|c|}{} & PORCENTAJE \\
\hline \multirow{7}{*}{} & Menos del 20\% & 18,8 \\
\cline { 2 - 3 } & Del 20 al 30\% & 5,1 \\
\cline { 2 - 3 } & Del 30 al 50\% & 6,9 \\
\cline { 2 - 3 } & Del 50 al 75\% & 9,1 \\
\cline { 2 - 3 } & Del 75 al 100\% & 12,7 \\
\cline { 2 - 3 } & Cero (0) & 7,3 \\
\cline { 2 - 3 } & Total & 59,9 \\
\hline Perdidos & Sistema & 40,1 \\
\hline Total & & 100,0 \\
\hline
\end{tabular}

Una evidencia más de lo anterior es la respuesta de los encuestados a la pregunta: “¿Gestiona su organización los documentos de exportación vía Internet?" en donde se encontró que un $24 \%$ de las empresas sí gestiona sus documentos por este medio y un $76 \%$ no lo hace. Lo anterior es reforzado con los resultados de: “¿Realiza su organización seguimiento por Internet de sus embarques?” en donde se encuentra lo siguiente:

Tabla 5. ¿Realiza su organización seguimiento por Internet de sus embarques?

\begin{tabular}{|l|l|c|}
\hline \multicolumn{2}{|c|}{} & PORCENTAJE \\
\hline \multirow{4}{*}{ Válidos } & Si & 21,4 \\
\cline { 2 - 3 } & No & 46,9 \\
\cline { 2 - 3 } & Total & 68,2 \\
\hline Perdidos & Sistema & 31,8 \\
\hline Total & 100,0 \\
\hline
\end{tabular}

La tabla anterior nos permite establecer el porcentaje de empresas que realiza 
seguimiento por Internet de sus embarques. Los resultados fueron que un 31,3\% de las empresas sí realizan este tipo de seguimiento a sus embarques mejorando así, la confiabilidad y la seguridad de estos, a diferencia de un $68,7 \%$ de las empresas encuestadas que no realizan el seguimiento por Internet de sus embarques.

\subsection{Interpretación de diferencias entre conglomerados}

Luego del anterior proceso, se filtró la base de datos posterior a que los estadísticos diseñados en SPSS para hallar conglomerados encontraren dos. Posteriormente, se procedió a realizar una comparación de medias para poblaciones independientes con el fin de hallar diferencias entre los dos grupos. Los resultados se describen a continuación:
El conglomerado 1 tiene un peso de $55 \%$ en la población, mientras que el 2 de $45 \%$, conteniendo 407 y 333 empresas respectivamente. Al hacer la comparación de medias, en ninguna de las variables demográficas ni de evaluación de nuevas tecnologías hubo diferencias estadísticamente significativas entre los dos conglomerados, lo que llevaría a concluir que el levantamiento de la información fue realizado en una población homogénea en dichos campos.

Alternativamente, en el conjunto de variables relativas a evaluación de Internet se encontraron diferencias significativas en las variables percepción de la influencia de Internet en la captación de nuevos clientes, porcentaje de uso de Internet en actividades de exportación y gestión documental de exportaciones vía Internet. A continuación las salidas de SPSS de acuerdo a cada una de las variables mencionadas:

Gráfica 2. Percepción de la Influencia de Internet en la captación de nuevos clientes.
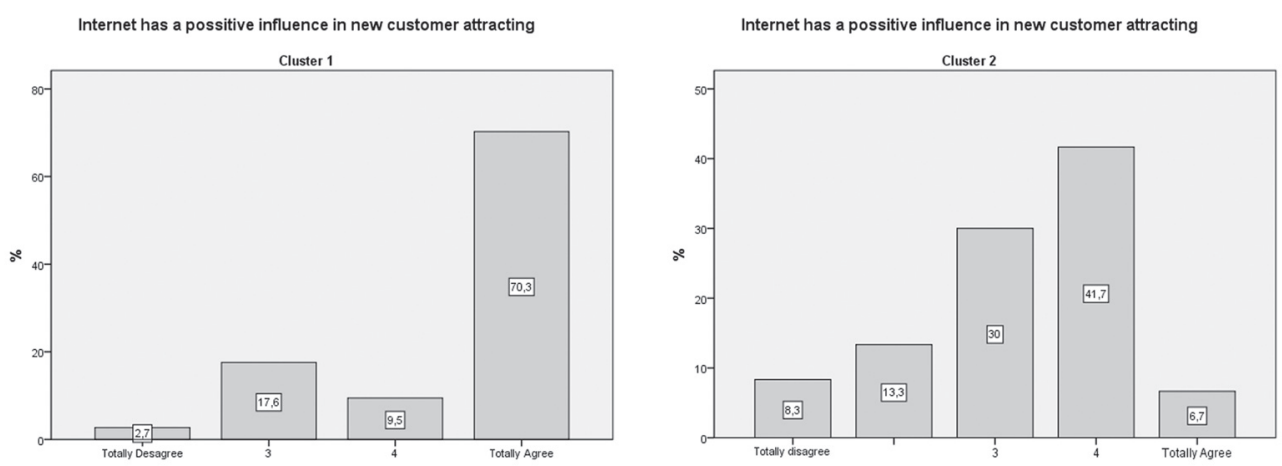
Gráfica 3. Porcentaje de uso de Internet en actividades de exportación.
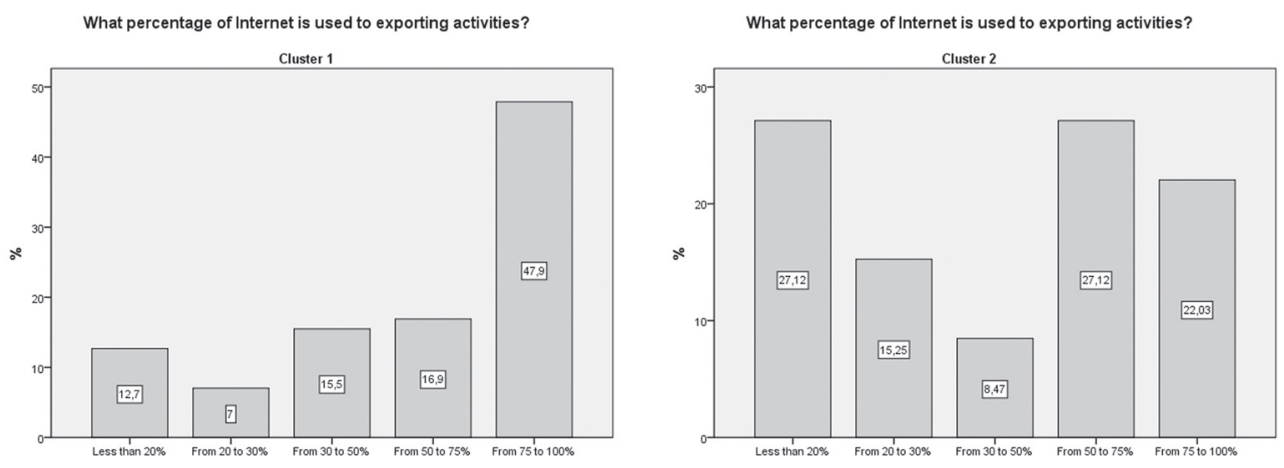

Gráfica 4. Gestión documental de exportaciones vía Internet.
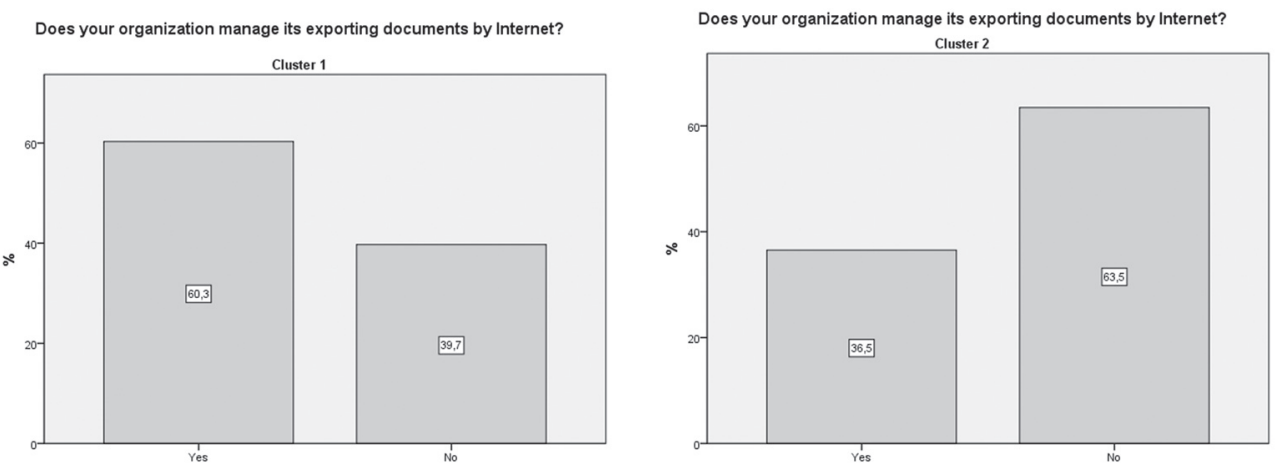

\section{Discusión}

A pesar de que las pequeñas compañías deberían aprovechar las ventajas del Internet para no estar embebidas en mercados locales (Yamin \& Sinkovics, 2006; Penot, 2010), es evidente el atraso en términos de apropiación tecnológica que ostentan las pequeñas y medianas compañías en Colombia. Realizando un ejercicio de comparación, mientras las grandes compañías del país están al nivel de "punta”, con respecto a éstas, las pequeñas tienen un atra-

$\curvearrowright \quad$ so significativo en dicho campo.

Aunado a lo anterior, el análisis clúster realizado, podría permitir nombrar el primer conglomerado como el "Adoptador temprano de nuevas tecnologías" $y$, el segundo, como el "Adoptador tardío de nuevas tecnologías". A pesar de que el primero es el de mayor peso en la población, $55 \%$ no es una mayoría significativa. Esta investigación se acerca a una comprensión de los niveles de apropiación tecnológica de las MiPymes colombianas. 
Futuras investigaciones deberían revisar poblaciones similares a las halladas por medio del análisis clúster con el fin de entender el riesgo que perciben algunas compañías y que les hace adoptadoras tardías. Se les propone revisar el planteamiento de Pezderka \& Sinkovics (2010). Lo anterior cobra importancia, dado que la carencia de evaluación y acción frente a dichos riesgos podría llevar a un atraso mayor al revelado en este trabajo.

\section{Bibliografía}

I. Acedo, F., \& Jones, M. (2007). Speed of internationalization and entrepreneurial cognition: Insights and a comparison between international new ventures exporters and domestic firms. Journal of World Business, 42, 236-252.

2. Acedo, F., Casillas, J., \& Moreno, A. (2008). La internacionalización de la empresa familiar: un análisis exploratorio. Management International, 12(3), 77-122.

3. Alcaldía Mayor de Bogotá. (s.f.). Resolución 320. Acto Administrativo.

4. Armario, J., Horrillo, M., \& Robles, E. (2009). La internacionalización de la empresa: el conocimiento experimental como derterminante del resultado en mercados exteriores. Cuadernos de Economia y Dirección de la Empresa, 39, 123-149.

5. Buckley, P. (1989). Foreing Direct Investment by Small Medium-Sized Enterprises: The Theoretical Background. Small Business Economics, 1(2), 89-100.

6. Cámara de Comercio de Bogotá;. (Extraído el 1 de diciembre de 2008, de http://camara.ccb.org.co/documen-
tos/636_2007_5_3_10_0_13_Inversion_Empresarial_2003_-_2005.pdf.). Dinámica de la inversión empresarial en Bogotá 2003-2005. Cámara de Comercio de Bogotá. Bogotá: Cámara de Comercio de Bogotá.

7. Camisón, C., \& López, A. (2010). Effect of SMEs' International Experience on Foreign Intensity and Economic Performance: The Mediating Role of Internationally Exploitable Assets and Competitive Strategy. Journal of Small Business Management, 48(2), 116-151.

8. Ching, H., \& Ellis, P. (2004). Marketing in ciberspace: What Factors drive ecommerce adoption? Journal of Marketing Management, 20(3,4), 409-429.

9. Comisión Intersectorial de Política Industrial. (Recuperado 2011-02-21 de http://www.cipi.gob.mx/html/principalesresultados.pdf). Principales Resultados del Observatorio PyME en México: Perfil del empresario. Comisión Intersectorial de Política Industrial. México D.F.: Comisión Intersectorial de Política Industrial.

io.De la Garza Toledo, E. (abril-junio de 1999). Relaciones laborales en las micro y pequeñas empresas en México. Papeles de Población(20), 175-196.

Ir.DNP, D. (2010). Reporte Global de Competitividad del Foro Económico Mundial 2010-2011: Resultados para Colombia - 2010. Bogotá.

I 2.Dunning, J. (2001). The Eclectic (oli) Paradigm of International Production; Past, Present and Future. International Journal of the Economics of Business, 8(2), 173-190.

I 3. Grant, R. (2004). Dirección estratégica, conceptos, técnicas y aplicaciones (Vol. 4). Valladolid: Thompson.

I 4. Interlat Group. (2008). Uso de Internet $y$ nuevas tecnologias como herramientas 
para las PYMES colombianas exportadoras o potencialmente exportadoras. Bogotá.

I 5.Martinez, F. (2009). Software libre y MiPymes. Vestigium (Número Especial), 37-50.

I6. Moen, O., \& Servais, P. (2002). Born Global or Gradual Global? Examining the Export Behavior of Small and Medium-Sized Enterprises. Journal of International Marketing, 10(3), 49-72.

I7. Moini, H., \& Tesar, G. (2005). The Internet and Internatiolization of Smaller Manufacturing Enterprises. Journal of Global Marketing, 18(3,4), 79-94.

I 8. Penot, J.-L. (2010). L'état et l'économie: l'évolution des paradigmes. Juridical Current, 13(2).

I9. Pezderka, N., \& Sinkovics, R. (2010). A Conceptualization of e-risk Perceptions and Implication for Small Firm Active Online Internationalization. International Business Review, Article in Press, 1-14.

20. Porter, M. (1983). Industrial Organization and the Evolution of Concepts for Strategic Planning: The New Learning. Managerial and Decision Economics, 4(3), 172-180.

2 I.Porter, M. (1991). Towards a Dynamic Theory of Strategy. Strategic Management Journal, 12, 95-117.

22.Porter, M. (2001). Strategy and the Internet. Hardvard Business Review, 79(3), 62-78.

23. Uribe, A., Rojas, S., \& Uribe, A. (2010). La dirección eestratégica y el cambio y su relacion con la internacionalizacion de empresas caso Pymes bogotanas. En XLV asamblea Cladea 2010, \& Cladea (ed.), XLV asamblea Cladea 2010 (Vol. XLV, págs. 1-25). Cartagena, Bolívar, Colombia: XLV asamblea Cladea 2010. 24. Vega, R., \& Rojas, S. (2009). Knowledge, Perception, and Application of Strategic Marketing in MSMEs (Micro, Small, and Medium Enterprises) in Bogotá. International Council For Small Business (ICSB) (págs. 1-27). Cincinati: International Council For Small Business (ICSB).

25.Velásquez, J., Fuentes, A., \& Jiménez, A. (2007). Mejoramiento de la gestión y uso de las TICs de las MiPymes y gobiernos locales a través de software libre. Universidad de Chile, Departamento de Ingeniería Industrial. Facultad de Ciencias Físicas y Matemáticas, Santiago de Chile.

26. Welsh, J., \& White, J. (1981). A Small Business is not a Little Big Business. Hardvard Business Review, 59(4), 18-27.

27. World Bank. (2003). Small and Medium Enterprises across the Globe: A New Database. Policy research working paper 3127.

28. Yamin, M., \& Sinkovics, R. (2006). Online Internationalisation, Physic Distiance Reduction and the Virtuality Trap. International Business Review, 15(4), 339-360. 